\title{
AN INVERSE PROBLEM FOR AN ABSORBING MEDIUM WITH MULTIPLE DISCONTINUITIES*
}

\author{
BY
}

\author{
ROBERT J. KRUEGER \\ University of Nebraska
}

1. Introduction. This paper deals with an inverse problem for wave propagation in an absorbing medium. Specifically, the problem we consider is a generalization of that considered in [5]. The motivation for the work in that paper came from a one-dimensional electromagnetic inverse scattering problem in which the conductivity $\sigma(z)$ and permittivity $\epsilon(z)$ of a slab of finite thickness situated between $z=0$ and $z=L$ were obtained by working in the space-time domain. The data required for the reconstruction of $\epsilon$ and $\sigma$ were determined by a portion of an incident plane wave and portions of the resulting reflected and transmitted waves. It was then shown that the actual reconstruction could be achieved by solving a single Gelfand-Levitan type integral equation.

The hypotheses used in [5] were motivated by the assumption that for $0<z<L$ the functions $\epsilon$ and $\sigma$ were continuous. In the present paper we relax this assumption by allowing $\epsilon$ and $\sigma$ to have any finite number of jump discontinuities on $0<z<L$, as well as being discontinuous at $z=0$ and $z=L$. Our solution of this problem is again carried out in the space-time domain. We show that the data for the solution can again be obtained from finite portions of a single incident, reflected and transmitted wave, although slightly "more" data are needed than in [5]. The reconstruction of $\epsilon$ and $\sigma$ can then be achieved by solving a linear integral equation containing advance and delay terms.

The problem we consider is modeled by

$$
u_{x x}-u_{t t}+A(x) u_{x}+B(x) u_{t}+C(x) u=0, \quad-\infty<x<\infty,-\infty<t<\infty
$$

where we assume there exist constants $x_{l}, 0=x_{0}<x_{1}<\cdots<x_{n}=l$ such that

(a) Support $A, B, C \subseteq[0, l]$.

(b) $A, B, C$ are piecewise continuous on $[0, l]$ with all discontinuities occurring at the points $x_{i}, i=0,1, \cdots, n$.

(c) $A^{\prime}$ and $B^{\prime}$ are continuous on the subintervals $\left(x_{i}, x_{i+1}\right), i=0,1, \cdots, n-1$.

(d) The solution $u(x, t)$ is everywhere continuous and piecewise $C^{2}$.

(e) There exist nonzero constants $c_{t}$ such that $c_{t} u_{x}\left(x_{i}+, t\right)=u_{x}\left(x_{i}-, t\right), i=0,1, \cdots$, $n$. (Notice that the quantity $c_{0}$ in this paper is equivalent to $c_{0}{ }^{-1}$ in [5].)

We assume that the coefficients $A, B, C$ of Eq. (1.1) (and therefore the location and number of discontinuities; i.e., the points $x_{i}$ and the integer $\left.n\right)$ are unknown on $(0, l)$, and seek to determine information about these coefficients by using information concerning

\footnotetext{
${ }^{*}$ Received January 10, 1978. A portion of this work was completed while the author was participating in the Air Force Systems Command University Resident Research Program at the Biometrics Division, School of Aerospace Medicine, Brooks AFB, Texas.
} 
the solution $u$ of $(1.1)$ in "free space", $x<0$ and $x>l$. Specifically, we have that a plane wave $u^{i}(x-t)$ propagating in the $+x$ direction gives rise to a reflected wave $u^{r}$ propagating in the $-x$ direction for $x<0$ and a transmitted wave $u^{t}$ propagating in the $+x$ direction for $x>l$. Thus, in the "free space" region, Eq. (1.1) has the solution

$$
\begin{aligned}
u(x, t) & =u^{i}(x-t)+u^{r}(x+t), & & x<0 \\
& =u^{t}(x-t) \quad, & & x>l .
\end{aligned}
$$

We require that $u^{i}(s)=u^{t}(s)=u^{r}(-s)=0$ for $s \geq 0$, and that these functions be continuous and piecewise $C^{2}$ (as mentioned in (d) above).

Now let us impose the additional hypotheses

(f) $u^{i \prime}(0-) \neq 0$.

(g) Let $S$ denote the set of all quantities $\left(x_{i}-x_{j}\right), 0 \leq j<i \leq n$. We assume that no element of $S$ can be expressed as a linear combination (with positive, integral coefficients) of other elements of $S$.

(h) A finite upper bound for $x_{n}$ is known.

We show in this paper that if hypotheses (a)-(h) are satisfied, then knowledge of the scattering data $u^{i}(s), u^{t}(s), u^{r}(-s)$ for $-4 l<s<0$ is sufficient to determine the coefficient $B(x)$ and the combination of coefficients $C-A^{\prime} / 2-A^{2} / 4$ on $(0, l)$, as well as the points $x_{i}$ and the integer $n$. The hypotheses (f)-(h) are imposed only to guarantee that the kernels of the reflection and transmission operators (see Sec. 3) and certain constants related to these operators can be determined. (This is elaborated in Sec. 4 and 5.) However, there are cases in which $(\mathrm{g})$ is not satisfied and these quantities can still be determined. (For example, let $n=3$ and $x_{0}=0, x_{1}=.15, x_{2}=.85, x_{3}=1$.)

If hypotheses (f)-(h) are not satisfied, the inverse problem is still solvable, but certain data may have to be obtained by means other than looking at scattered waves. In particular, the synthesis problem is still solvable. In other problems, such as biological applications, the number and location (or approximate location) of the $x_{i}$ 's is known and so hypothesis $(\mathrm{h})$ can be dropped and it may be possible to relax $(\mathrm{g})$, depending on the geometry.

As mentioned above, the motivation for our work comes from an electromagnetic scattering problem. To see the connection between this and Eq. (1.1), consider an electromagnetic wave propagating along the $z$ axis normal to the slab. The transverse electric field $E(z, t)$ satisfies

$$
E_{z z}-\epsilon(z) \mu_{0} E_{t t}-\sigma(z) \mu_{0} E_{t}=0, \quad-\infty<z<\infty,-\infty<t<\infty,
$$

where $\mu_{0}$ is constant and $\epsilon$ and $\sigma$ have jump discontinuities at the points $z_{i}$, where $0=z_{0}<$ $z_{1}<\cdots<z_{n}=L$. As shown in [5], the change of variable

$$
x=\int_{0}^{z}\left[\epsilon(s) \mu_{0}\right]^{1 / 2} d s, \quad x\left(z_{i}\right)=x_{i}, u(x, t)=E(z, t)
$$

reduces (1.2) to the form (1.1) with

$$
c_{i}=\left[\epsilon\left(z_{i}+\right) / \epsilon\left(z_{i}-\right)\right]^{1 / 2} .
$$

The solution of inverse wave propagation problems in absorbing media has been studied by several authors. The work of Buzdin [1] is concerned with a half-space problem, but results in a system of seven coupled nonlinear integrodifferential equations. 
Jaulent [2] considers both one-dimensional and radial inverse problems in absorbing media by means of a steady-state approach. Under certain hypotheses the solution of the inverse problem then reduces to the solution of two sets of dual linear integral equations and a nonlinear initial value problem. Weston $[8,9]$ and Weston and Krueger [10] consider a one-dimensional slab problem (using a space-time technique) whose solution is obtained from a dual set of linear integral equations. In all of the above problems, however, the hypotheses imposed imply that the properties of the absorbing medium must vary smoothly in the spatial variable. In other words, when writing these problems in the form (1.1), the coefficients $A$ and $B$ are $C^{1}$ for $-\infty<x<\infty$ and $C$ is continuous for $-\infty<$ $x<\infty$. Such conditions are quite restrictive since they do not allow for the fact that the interface between two different media is characterized by discontinuities in $A, B, C$.

Of course, considerable analysis has been done for the case $A \equiv B \equiv 0, C$ piecewise continuous. (For example, see Roseau [7] for a time-dependent approach, and Kay [4] for a steady-state approach.) However, these analyses do not take into account the jump discontinuities in $u_{x}$ given in (e) and consequently are not applicable to certain types of problems, such as electromagnetic inverse problems using a fixed angle of incidence. Therefore, as was brought out in [5, Sec. 2], the results presented here also have application to some unresolved inverse scattering problems in nondissipative media.

The outline of this paper is as follows: In Sec. 2 we generalize the weak Riemann function developed in [5] to allow for multiple jump discontinuities. Using this function we can then express the solution $u$ of (1.1) in terms of the waves $u^{i}$ and $u^{r}$, or $u^{t}$. Sec. 3 is concerned with the development of the scattering operators for (1.1). In Sec. 4 an analogue of the Gelfand-Levitan equation is developed. This equation contains, in general, both advance and delay terms and its solution yields the coefficient $B$ and the combination $C-$ $A^{\prime} / 2-A^{2} / 4$. We also present a numerical method of solution. Sec. 5 is concerned with how certain data for the solution of the inverse problem can be obtained. Finally, Sec. 6 deals with the special case of an electromagnetic inverse problem in which the conductivity and permittivity are piecewise constant functions.

2. The weak Riemann function. As in [5] we begin by solving the Cauchy problem for (1.1) with the data being $u(x, t), u_{x}(x, t)$ with $x=0+$ or $x=l-$. In this case we use a generalization of the weak Riemann function developed in [5]. Letting $\xi=x+t, \eta=x-t$, we require that the weak Riemann function $g\left(\xi, \eta ; \xi_{0}, \eta_{0}\right)$ now satisfy

(a) $L^{*} g=0$ for $\xi+\eta \neq 2 x_{i}, i=0,1, \cdots, n$ (where $L^{*}$ is the formal adjoint of $L$ );

(b) $g$ is a continuous function of $\xi, \eta, \xi_{0}, \eta_{0}$;

(c) $g\left(\xi_{0}, \eta ; \xi_{0}, \eta_{0}\right)=\exp \left\{\frac{1}{2} \int_{\left(\xi_{0}+\eta_{0}\right) / 2}^{\left(\xi_{0}+\eta\right) / 2}(A(s)+B(s)) d s\right\}$;

(d) $g\left(\xi, \eta_{0} ; \xi_{0}, \eta_{0}\right)=\exp \left\{\frac{1}{2} \int_{\left(\xi_{0}+\eta_{0}\right) / 2}^{\left(\xi+\eta_{0}\right) / 2}(A(s)-B(s)) d s\right\}$;

(e) $\left[g_{\xi}+g_{\eta}\right]^{\xi+\eta=2 x_{i}+}{ }_{\xi+\eta=2 x_{i-}}=\left.[A]^{2 x_{i}{ }^{+}{ }_{2 x_{i}} g}\right|_{\xi+\eta=2 x_{i}}$ for $i=0,1, \cdots, n$, where we denote differences in function values with $[f]_{P}^{Q}=f(P)$.

It is clear that such a function exists and is unique.

We begin by expressing the solution $u$ of (1.1) in terms of Cauchy data on $x=l-$. In what follows, $\theta$ is the Heaviside function, $\theta(s)=0$ if $s \leq 0, \theta(s)=1$ if $s>0$. 
Lemma 1. For $x<l$ and any $t$ we have

$$
\begin{aligned}
2 u(x, t) & =\left.g u\right|_{P}+\left.g u\right|_{Q}+\sum_{i=0}^{n-1} \theta\left(x_{i}-x\right)\left(c_{i}-1\right) \int_{R_{l}}^{S_{i}} g u_{x} d s \\
& +\int_{P}^{Q}\left\{u\left(g_{\xi}+g_{\eta}\right)-u_{x} g-A(l-) u g\right\} d s
\end{aligned}
$$

where $\xi_{0}=x+t, \eta_{0}=x-t, P=(l-, t+x-l), Q=(l-, t-x+l), R_{i}=\left(x_{i}+, t-x+\right.$ $\left.x_{i}\right), S_{i}=\left(x_{i}+, t+x-x_{i}\right)$. The integrals under the summation sign are along the lines $x=$ $x_{i}+$ and the last integral on the right-hand side is along the line $x=l-$.

Proof: To fix our ideas, consider a given point $\left(\xi_{0}, \eta_{0}\right)$. We integrate the expression $g L u-u L^{*} g=0$ over all regions in the $(\xi, \eta)$ plane (bounded by the characteristics through $\left(\xi_{0}, \eta_{0}\right)$ and the line $\left.\xi+\eta=2 l-\right)$ in which the expression is continuously differentiable. In general, these regions are bounded by the lines $\xi+\eta=2 x_{i}$ and also by the characteristics $\xi$ $=2 x_{i}-\eta_{0}$ and $\eta=2 x_{i}-\xi_{0}$ along which the discontinuities in $g_{\xi}$ and $g_{\eta}$ are propagated respectively. Applying Green's formula and adding the resulting integrals, we find that the properties of the weak Riemann function cause the integrals along the lines $\xi+\eta=2 x_{i}$ to reduce to the summation of integrals on the right-hand side of (2.1) and the integrals along the characteristics $\xi=2 x_{i}-\eta_{0}, \eta=2 x_{i}-\xi_{0}$ cancel out. Thus we arrive at (2.1) and the proof is completed. (Notice that the integral on the right-hand side of (2.1) along $x=l$ - is actually composed of a sum of integrals along $x=l-$, the integrand in each single integral being continuously differentiable.)

We now introduce operators $\mathfrak{K}_{i}$ and $\mathfrak{K}_{i}$ which will be used to trace internal reflections in the slab. If $v$ is any function of $x \pm t$, we define the shift operator $\mathcal{F C}_{t}$ by

$$
\mathcal{H}_{i} v(x \pm t)=\frac{1}{2}\left(c_{i}+1\right) v(x \pm t)-\frac{1}{2}\left(c_{i}-1\right) v\left(2 x_{i}-x \pm t\right) \exp \left\{\mp \int_{x}^{x_{i}} B(s) d s\right\},
$$

taking either the upper or lower signs throughout. Furthermore, we require that for any functions of $x$, say $f$ and $g$, we have

$$
\mathfrak{H}_{i}[f(x) v(x \pm t)+g(x) w(x \pm t)]=f(x) \mathfrak{H}_{i} v(x \pm t)+g(x) \mathfrak{H}_{i} w(x \pm t)
$$

We define the linear operator $\mathfrak{K}_{i}$ similarly, with

$$
\mathcal{K}_{i} v(x \pm t)=\frac{1}{2}\left(c_{i}+1\right) v(x \pm t)+\frac{1}{2}\left(c_{i}-1\right) v\left(2 x_{i}-x \pm t\right) \exp \left\{\mp \int_{x}^{x_{i}} B(s) d s\right\} .
$$

We define composition of these operators in the obvious way,

$$
\left(\mathfrak{H}_{i} \circ \mathfrak{H}_{j}\right) v(x \pm t)=\mathfrak{H}_{i}\left(\mathfrak{H}_{j} v(x \pm t)\right) \equiv \mathfrak{H}_{i} \circ \mathfrak{H}_{j} v(x \pm t) .
$$

Observe that if we let $\mathscr{G}$ denote the identity operator,

$$
\mathfrak{I} f(x) v(x \pm t)=f(x) v(x \pm t)
$$

then we have

LEMMA 2. $c_{i}^{-1} \mathfrak{K}_{i} \circ \mathfrak{K C}_{i}=c_{i}^{-1} \mathfrak{H}_{i} \circ \mathfrak{K}_{i}=\mathscr{J}$.

Proof:

$$
\mathcal{K}_{i} \circ \mathcal{K}_{i} v(x \pm t)=\frac{1}{2}\left(c_{i}+1\right) \mathcal{K}_{i} v(x \pm t)
$$




$$
\begin{aligned}
& -\frac{1}{2}\left(c_{i}-1\right) \exp \left\{\mp \int_{x}^{x_{i}} B(s) d s\right\} \mathcal{K}_{i} v\left(2 x_{i}-x \pm t\right) \\
& =\frac{1}{4}\left(c_{i}+1\right)^{2} v(x \pm t)-\frac{1}{4}\left(c_{i}-1\right)^{2} v(x \pm t) \\
& =c_{i} v(x \pm t) .
\end{aligned}
$$

Clearly, a similar result holds for $\mathfrak{H}_{i} \circ \mathfrak{K}_{i}$, and the proof is completed.

Now for any integer $k, 1 \leq k \leq n$, let $J(k)$ denote the set of all sequences $j=\left(j_{k}, j_{k+1}\right.$, $\cdots, j_{n}$ ) such that:

(a) Each $j_{i}$ is either $-1,0$ or +1 ;

(b) The last nonzero $j_{i}$ in any sequence is 1 ;

(c) The nonzero entries in the sequence $\left(j_{k}, j_{k+1}, \cdots, j_{n}\right)$ alternate in sign.

Notice that if we set

$$
J(k, 0)=\left\{j \in J(k): \sum_{i=k}^{n} j_{i}=0\right\}
$$

and

$$
J(k, 1)=\left\{j \in J(k): \sum_{i=k}^{n} j_{i}=1\right\}
$$

then

$$
J(k)=J(k, 0) \cup J(k, 1)
$$

Now for $j \in J(k)$ set

$$
r_{j}=2 \sum_{i=k}^{n} j_{i} x_{i}, \quad C_{j}^{ \pm}=p_{k} \prod_{i=k}^{n}\left(g_{i}\right)^{\left|j_{i}\right|} G\left(x_{i}\right)^{\mp j_{i}}
$$

where

$$
p_{k}=2^{k-n-1} \prod_{i=k}^{n}\left(c_{i}+1\right), \quad g_{i}=\left(c_{i}-1\right) /\left(c_{i}+1\right), \quad G(x)=\exp \left\{-\int_{0}^{x} B(s) d s\right\} .
$$

It can now be shown that

$$
\mathfrak{H}_{k} \circ \mathfrak{H C}_{k+1} \circ \cdots \circ \mathfrak{H}_{n} v(x \pm t)=\sum_{j \in J(k, 0)} C_{j}^{\mp} v\left(r_{j}+x \pm t\right)-G(x)^{\mp 1} \sum_{j \in J(k, 1)} C_{j}^{\mp} v\left(r_{j}-x \pm t\right)
$$

taking either the upper or lower signs throughout. The expressions for $\mathcal{K}_{k} \circ \mathfrak{K}_{k+1} \circ \cdots \circ$ $\mathfrak{K}_{n} v(x \pm t)$ are similar to (2.2), except that the minus sign between the two summations is replaced with a plus sign.

In an analogous manner we define $\tilde{J}(k)$ to be the set of all sequences $j=\left(j_{0}, j_{1}, \cdots, j_{k}\right)$ such that:

(a) Each $j_{i}$ is either $-1,0$ or +1 ;

(b) The first nonzero $j_{i}$ in any sequence is 1 ;

(c) The nonzero entries in the sequence alternate in sign. 
Now set

$$
\tilde{J}(k, 0)=\left\{j \in \tilde{J}(k): \sum_{i=0}^{k} j_{i}=0\right\}, \quad \tilde{J}(k, 1)=\left\{j \in \tilde{J}(k): \sum_{i=0}^{k} j_{i}=1\right\},
$$

and, for $j \in \tilde{J}(k)$,

$$
\tilde{r}_{j}=2 \sum_{i=0}^{k} j_{i} x_{i}, \quad \tilde{C}_{j}^{ \pm}=\tilde{p}_{k} \prod_{i=0}^{k}\left(g_{i}\right)^{\left|j_{i}\right|} G\left(x_{i}\right)^{\mp j_{i}}
$$

where

$$
\tilde{p}_{k}=2^{-k-1} \prod_{i=0}^{k}\left(c_{i}+1\right)
$$

It follows that

$\mathcal{K}_{k} \circ \mathcal{K}_{k-1} \circ \cdots \circ \mathcal{K}_{0} v(x \pm t)=\sum_{J \in J(k, 0)} \tilde{C}_{j}{ }^{\mp} v\left(\tilde{r}_{j}+x \pm t\right)+G(x)^{\mp 1} \sum_{j \in \tilde{J}(k, 1)} \tilde{C}_{j}{ }^{\mp} v\left(\tilde{r}_{j}-x \pm t\right)$.

We now show that it is possible to express the solution $u$ of $(1.1)$ in terms of right and left moving waves at $x=l$. In the statement of the theorem, we assume $k$ is an integer, $1 \leq$ $k \leq n$, and $D_{ \pm}(x)=C(x)-\frac{1}{2} A^{\prime}(x) \pm \frac{1}{2} B^{\prime}(x)+\frac{1}{4}\left(B^{2}(x)-A^{2}(x)\right)$.

TheOREM 1. Assume the Cauchy data for Eq. (1.1) is

$$
\begin{gathered}
u(l, t)=v(l-t)+w(l+t) \\
u_{x}(l, t)=c_{n} v^{\prime}(l-t)+c_{n} w^{\prime}(l+t)
\end{gathered}
$$

where $v$ and $w$ are continuous, piecewise $C^{2}$ functions. Then there exist piecewise continuously differentiable functions $M_{ \pm}{ }^{k}(x, y, l)$ defined for $x<x_{k}, x \leq y \leq 2 l-x$ such that if $x_{k-1}<x<x_{k}$, then

$$
\begin{aligned}
u(x, t)= & \exp \left\{\frac{1}{2} \int_{x}^{l}(A(s)-B(s)) d s\right\}\left\{\mathcal{H}_{k} \circ \mathcal{H}_{k+1} \circ \cdots \circ \mathcal{H}_{n} v(x-t)\right. \\
& \left.+\int_{x}^{2 l-x} v(y-t) M_{+}{ }^{k}(x, y, l) d y\right\} \\
& +\exp \left\{\frac{1}{2} \int_{x}^{l}(A(s)+B(s)) d s\right\}\left\{\mathcal{C}_{k} \circ \mathcal{H}_{k+1} \circ \cdots \circ \mathcal{H}_{n} w(x+t)\right. \\
& \left.+\int_{x}^{2 l-x} w(y+t) M_{-}{ }^{k}(x, y, l) d y\right\} .
\end{aligned}
$$

Furthermore,

$$
\frac{d}{d x} M_{+}^{k}(x, x, l)=\frac{1}{2} p_{k} D_{+}(x), \quad x_{k-1}<x<x_{k}
$$

Proof: In the same manner as in [5, Lemmas 3 and 4] it can be shown that functions $K_{ \pm}\left(x, y, x_{i}\right), L_{ \pm}\left(x, y, x_{i}\right)$ can be defined by the following relations:

$$
K_{+}\left(x, y, x_{i}\right) \exp \left\{\frac{1}{2} \int_{x}^{x_{i}}(A(s)-B(s)) d s\right\}=g_{\eta}\left(2 x_{i}-y+t, y-t ; x+t, x-t\right)
$$




$$
\begin{aligned}
& K_{-}\left(x, y, x_{i}\right) \exp \left\{\frac{1}{2} \int_{x}^{x_{i}}(A(s)+B(s)) d s\right\}=g_{\xi}\left(y+t, 2 x_{i}-y-t ; x+t, x-t\right) \\
& L_{+}\left(x, y, x_{i}\right) \exp \left\{\frac{1}{2} \int_{x}^{x_{i}}(A(s)-B(s)) d s\right\}=g\left(2 x_{i}-y+t, y-t ; x+t, x-t\right) \\
& L_{-}\left(x, y, x_{i}\right) \exp \left\{\frac{1}{2} \int_{x}^{x_{i}}(A(s)+B(s)) d s\right\}=g\left(y+t, 2 x_{i}-y-t ; x+t, x-t\right) .
\end{aligned}
$$

These functions satisfy the following conditions:

$$
\begin{gathered}
K_{ \pm}\left(x, 2 x_{i}-x, x_{i}\right)=\left.\frac{1}{4}(A \pm B)\right|_{x_{i^{-}}} \exp \left\{ \pm \int_{x}^{x_{i}} B(s) d s\right\}, x_{i-1}<x<x_{i}, \\
\frac{d}{d x} K_{ \pm}\left(x, x, x_{i}\right)=\frac{1}{2} D_{ \pm}(x), x \neq x_{j}, 1 \leq j \leq n, \\
L_{ \pm}\left(x, 2 x_{i}-x, x_{i}\right)=\exp \left\{ \pm \int_{x}^{x_{i}} B(s) d s\right\}, \\
L_{ \pm}\left(x, x, x_{i}\right)=1 .
\end{gathered}
$$

We now show that for any integer $k, 0 \leq k \leq n$,

$$
\begin{aligned}
& \frac{1}{2}\left[\left.g u\right|_{P}+\left.g u\right|_{Q}+\sum_{i=k}^{n-1}\left(c_{i}-1\right) \int_{R_{i}}^{s_{i}} g u_{x} d s+\int_{P}^{Q}\left\{u\left(g_{\xi}+g_{\eta}\right)-u_{x} g-A(l-) u g\right\} d s\right] \\
& =\exp \left\{\frac{1}{2} \int_{x}^{l}(A(s)-B(s)) d s\right\}\left\{\mathcal{K}_{k} \circ \cdots \circ \mathfrak{K}_{n} v(x-t)\right. \\
& \left.+\int_{x}^{2 l-x} v(y-t) M_{+}^{k}(x, y, l) d y\right\}+\exp \left\{\frac{1}{2} \int_{x}^{l}(A(s)+B(s)) d s\right\} \\
& \left\{\mathfrak{H C}_{k} \circ \cdots \circ \mathcal{H}_{n} w(x+t)+\int_{x}^{2 l-x} w(y+t) M_{-}{ }^{k}(x, y, l) d y\right\}
\end{aligned}
$$

where $M_{+}{ }^{k}(x, x, l)$ satisfies (2.4). (If $k=n$, the summation on the left-hand side does not appear). To simplify matters in what follows, we exploit the linearity of (1.1) by assuming $w \equiv 0$. Clearly we can likewise consider the case $v \equiv 0, w \neq \equiv 0$ and add the results to obtain our desired conclusion.

To verify (2.5), we proceed by induction on the number of discontinuities considered (i.e., the number of terms included in the summation on the left-hand side of (2.5)). The case $k=n$ has already been shown in [5, Theorem 1]. (Notice that the quantity $N_{+}(x, y, l)$ in [5] is equal to $2 M_{+}{ }^{n}(x, y, l)$.) Thus, assuming (2.5) is valid for $k=i+1$, we now consider $k \equiv$. Our induction hypothesis implies that $u(x, t)$ is given by $(2.3)$ for $x>x_{i}$ by setting $k=i+1$. Using (2.3) to express $u_{x}\left(x_{j}+, t\right)$ (making use of the remark following the proof of Lemma 1), we now form the integral

$$
\int_{t-x+x_{i}}^{t+x-x_{i}} g\left(x_{i}+s, x_{i}-s ; x+t, x-t\right) u_{x}\left(x_{i}+, s\right) d s
$$

and integrate by parts the terms involving $v^{\prime}$. This gives

$$
\int_{R_{i}}^{s_{i}} g u_{x} d s=\exp \left\{\frac{1}{2} \int_{x}^{l}(A(s)-B(s)) d s\right\}\left[\sum_{j \in J(i+1,0)} C_{j}^{+} v\left(r_{j}+x-t\right)\right.
$$




$$
\begin{aligned}
& +G\left(x_{i}\right) \sum_{j \in J(i+1,1)} C_{j}^{+} v\left(r_{j}-2 x_{i}+x-t\right)-G(x) G\left(x_{i}\right)^{-1}\left\{\sum_{j \in J(i+1,0)} C_{j}{ }^{+} v\left(r_{j}+2 x_{i}-x-t\right)\right. \\
& \left.+G\left(x_{i}\right) \sum_{j \in J(i+1,1)} C_{j}{ }^{+} v\left(r_{j}-x-t\right)\right\}+\sum_{j \in J(i+1,0)} C_{j}{ }^{+} \int_{r_{j}+x}^{r_{j}+2 x_{i}-x} v(y-t)\left\{K_{+}\left(x, y-r_{j}, x_{i}\right)\right. \\
& \left.-G(x) G\left(x_{i}\right)^{-1} K_{-}\left(x, r_{j}+2 x_{i}-y, x_{i}\right)-\frac{1}{2}\left(A\left(x_{i}+\right)-B\left(x_{i}+\right)\right) L_{+}\left(x, y-r_{j}, x_{i}\right)\right\} d y \\
& +G\left(x_{i}\right) \sum_{j \in J(i+1,1)} C_{j}^{+} \int_{r_{j}-2 x_{i}+x}^{r_{j}-x} v(y-t)\left\{K_{+}\left(x, y-r_{j}+2 x_{i}, x_{i}\right)\right. \\
& \left.-G(x) G\left(x_{i}\right)^{-1} K_{-}\left(x, r_{j}-y, x_{i}\right)+\frac{1}{2}\left(A\left(x_{i}+\right)+B\left(x_{i}+\right)\right) G(x) G\left(x_{i}\right)^{-1} L_{-}\left(x, r_{j}-y, x_{i}\right)\right\} d y \\
& -M_{+}{ }^{i+1}\left(x_{i}+, x_{i}+, l\right) \int_{x}^{2 x_{i}-x} v(y-t) L_{+}\left(x, y, x_{i}\right) d y \\
& \left.\cdot \int_{2 l-2 x_{i}+x}^{2 l-x} v(y-t) L_{-}\left(x, 2 l-y, x_{i}\right) d y+\int_{x}^{2 i-x} v(y-t) Z^{i+1}(x, y, l) d y\right]
\end{aligned}
$$

where $Z^{i+1}(x, y, l)$ is a sum of integrals, and $Z^{i+1}(x, x, l) \equiv 0$.

To complete the induction step, multiply (2.6) by $\frac{1}{2}\left(c_{i}-1\right)$ and add to (2.5) with $k=$ $i+1$. The terms on the right-hand side of the resulting expression which do not involve integrals sum up to $\mathcal{H}_{i} \circ \mathcal{H}_{i+1} \circ \cdots \circ \mathcal{H}_{n} v(x-t)$. The remaining terms can be written as and add to (2.5) with $k=i+1$. The terms on the right-hand side of the resulting expression which do not involve integrals sum up to $\mathfrak{H}_{i} \circ \mathfrak{H}_{i+1} \circ \cdots \circ \mathfrak{H}_{n} v(x-t)$. The remaining terms can be written as

$$
\int_{x}^{2 l-x} v(y-t) M_{+}^{i}(x, y, l) d y
$$

where

$$
\begin{aligned}
\frac{d}{d x} M_{+}{ }^{i}(x, x, l) & =\frac{d}{d x} M_{+}{ }^{i+1}(x, x, l) \\
& +\frac{1}{2}\left(c_{i}-1\right) \frac{d}{d x}\left[p_{i+1} K_{+}\left(x, x, x_{i}\right)-p_{i+1} \exp \left\{\int_{x}^{x_{i}} B(s) d s\right\} K_{-}\left(x, 2 x_{i}-x, x_{i}\right.\right. \\
& -\frac{1}{2} p_{i+1}\left(A\left(x_{i}+\right)-B\left(x_{i}+\right)\right) L_{+}\left(x, x, x_{i}\right)-M_{+}{ }^{i+1}\left(x_{i}+, x_{i}+, l\right) L_{+}\left(x, x, x_{i}\right) \\
& \left.+Z^{i+1}(x, x, l)\right]=\frac{1}{2} p_{i} D_{+}(x), \quad x_{i-1}<x<x_{i} .
\end{aligned}
$$

Hence, Eq. (2.5) is justified, and the proof of the theorem now follows from Lemma 1.

Corollary 1. Assume the Cauchy data for Eq. (1.1) is

$$
u(0, t)=v(-t)+w(t), \quad u_{x}(0, t)=c_{0}{ }^{-1} v^{\prime}(-t)+c_{0}{ }^{-1} w^{\prime}(t)
$$

where $v$ and $w$ are continuous, piecewise $C^{2}$ functions. Then there exist piecewise continu- 
ously differentiable functions $M_{ \pm}{ }^{k}(x, y, 0)$ defined for $x>x_{k},-x \leq y \leq x$ such that if $x_{k}$ $<x<x_{i+1}$, then

$$
\begin{aligned}
u(x, t) & =\exp \left\{\frac{1}{2} \int_{x}^{0}(A(s)-B(s)) d s\right\}\left\{\mathcal{K}_{k} \circ \mathcal{K}_{k-1} \circ \cdots \circ \mathfrak{K}_{0} v(x-t)\right. \\
& \left.-\int_{-x}^{x} v(y-t) M_{+}{ }^{k}(x, y, 0) d y\right\}+\exp \left\{\frac{1}{2} \int_{x}^{0}(A(s)+B(s)) d s\right\} \\
& \left\{\mathcal{K}_{k} \circ \mathcal{K}_{k-1} \circ \cdots \circ \mathfrak{K}_{0} w(x+t)-\int_{-x}^{x} w(y+t) M_{-}^{k}(x, y, 0) d y\right\} .
\end{aligned}
$$

Furthermore,

$$
\frac{d}{d x} M_{-}^{k}(x, x, 0)=\frac{1}{2} q_{k} D_{-}(x), \quad x_{k}<x<x_{k+1}
$$

where

$$
q_{k}=2^{-k-1} \prod_{i=0}^{k}\left(c_{i}^{-1}+1\right) .
$$

Proof: Using the change of variables $\zeta=l-x, \tau=t+l$ and $u(x, t)=\tilde{u}(\zeta, \tau)$ we convert the given problem to one in which data is given on $\zeta=l$, namely,

$$
\tilde{u}_{\zeta \zeta}-\tilde{u}_{\tau \tau}+\tilde{A}(\zeta) \tilde{u}_{\zeta}+\tilde{B}(\zeta) \tilde{u}_{\tau}+\tilde{C}(\zeta) \tilde{u}=0
$$

where $\tilde{A}(\zeta)=-A(x), \tilde{B}(\zeta)=B(x)$ and $\tilde{C}(\zeta)=C(x)$. The data itself is obtained from

$$
\begin{gathered}
v(x-t)=v(2 l-\zeta-\tau)=\tilde{w}(\zeta+\tau), \\
w(x+t)=w(\tau-\zeta)=\tilde{v}(\zeta-\tau) .
\end{gathered}
$$

Finally, the $\zeta$ derivative of $\tilde{u}$ has jump discontinuities at $\zeta_{i}=l-x_{n-i}, 0 \leq i \leq n$, given by $\tilde{u}_{\zeta}\left(\zeta_{i}-, \tau\right)=\tilde{c}_{i} \tilde{u}_{\zeta}\left(\zeta_{i}+, \tau\right)$ where $\tilde{c}_{i}=c_{n-i}{ }^{-1}, 0 \leq i \leq n$. After applying Theorem 1 to this new problem, we obtain our result by converting back to $(x, t)$ coordinates.

3. The scattering operators. The scattering operators for Eq. (1.1) map any incident wave $u^{i}$ satisfying the hypotheses given in Sec. 1 into the corresponding reflected and transmitted waves. To simplify our expressions for these operators, we introduce some notation. Let $\mathcal{J C}^{r}, \mathcal{H}^{i}$ denote shift operators defined by

$$
\begin{aligned}
& \mathcal{H C}^{r} v(-t)=\left.\frac{1}{2} a_{-}\left\{\mathcal{H}_{1} \circ \mathfrak{H}_{2} \circ \cdots \circ \mathcal{H}_{n} v(x-t)-c_{0} \mathcal{K}_{1} \circ \mathfrak{K}_{2} \circ \cdots \circ \mathfrak{K}_{n} v(x-t)\right\}\right|_{x=0}, \\
& \mathfrak{H C}^{i} v(-t)=\left.\frac{1}{2} a_{-}\left\{\mathcal{H}_{1} \circ \mathfrak{H}_{2} \circ \cdots \circ \mathfrak{K}_{n} v(x-t)+c_{0} \mathcal{K}_{1} \circ \mathfrak{K}_{2} \circ \cdots \circ \mathfrak{K}_{n} v(x-t)\right\}\right|_{x=0}
\end{aligned}
$$

where

$$
a_{ \pm}=\exp \left\{\frac{1}{2} \int_{0}^{l}(A(s) \pm B(s)) d s\right\}
$$

Thus, for any $s$ we write

$$
\begin{aligned}
& \operatorname{JC}^{r} v(s)=-\frac{1}{2} a_{-}\left\{\left(c_{0}-1\right) \sum_{j \in J(1,0)} C_{j}^{+} v\left(r_{j}+s\right)+\left(c_{0}+1\right) \sum_{j \in J(1,1)} C_{j}^{+} v\left(r_{j}+s\right)\right\}, \\
& \mathcal{J C}^{i} v(s)=\frac{1}{2} a_{-}\left\{\left(c_{0}+1\right) \sum_{j \in J(1,0)} C_{j}^{+} v\left(r_{j}+s\right)+\left(c_{0}-1\right) \sum_{j \in J(1,1)} C_{j}^{+} v\left(r_{j}+s\right)\right\} .
\end{aligned}
$$


THEOREM 2. There exist unique piecewise continuous functions $V$ and $W$ such that

$$
\begin{array}{cc}
u^{r}(t)=\mathcal{F C}^{r} u^{t}(-t)+\int_{-t}^{0} V(s+t) u^{t}(s) d s, & t>0, \\
u^{i}(t)=\mathfrak{F}^{i} u^{t}(t)+\int_{t}^{0} W(t-s) u^{t}(s) d s, & t<0 .
\end{array}
$$

Proof: For $0<x<x_{1}$ Theorem 1 gives us

$$
\begin{aligned}
u(x, t) & =\exp \left\{\frac{1}{2} \int_{x}^{l}(A(s)-B(s)) d s\right\}\left\{\mathcal{C}_{1} \circ \mathfrak{H}_{2} \circ \cdots \circ \mathcal{F}_{n} u^{t}(x-t)\right. \\
& \left.+\int_{x}^{2 l-x} u^{t}(y-t) M_{+}{ }^{1}(x, y, l) d y\right\} .
\end{aligned}
$$

Setting $x=0+$, we obtain

$$
\begin{aligned}
u^{i}(-t)+u^{r}(t)= & a_{-}\left\{\left.\left(\mathcal{H C}_{1} \circ \mathcal{F}_{2} \circ \cdots \circ \mathcal{H}_{n} u^{t}(x-t)\right)\right|_{x=0}\right. \\
& \left.+\int_{-t}^{2 l-t} u^{t}(s) M_{+}{ }^{1}(0, s+t, l) d s\right\} .
\end{aligned}
$$

Now differentiate (3.5) with respect to $x$, set $x=0+$ and integrate from 0 to $t$ (using the fact that $u^{i}, u^{r}, u^{t}$ are continuous and piecewise $C^{2}$ ) to get

$$
\begin{aligned}
-u^{i}(-t)+u^{r}(t)= & c_{0} a_{-}\left\{-\left.\left(\mathcal{K}_{1} \circ \mathfrak{K}_{2} \circ \cdots \circ \mathcal{K}_{n} u^{t}(x-t)\right)\right|_{x=0}\right. \\
& \left.+\int_{-t}^{0} u^{t}(s) V_{1}(s+t) d s\right\}
\end{aligned}
$$

where $V_{1}$ is piecewise continuous. The theorem now follows from (3.6) and (3.7).

By inverting (3.4) (i.e., by solving for $u^{t}$ in terms of $u^{i}$ ) we obtain the forward scattering operator or transmission operator. Before this can be done, we need some additional notation. We put the elements of $J(1,0)$ in a one-to-one correspondence with the first $2^{n-1}$ nonnegative integers, $0,1, \cdots, 2^{n-1}-1$ with the sequence $(0,0, \cdots, 0)$ corresponding to $0:(0,0, \cdots, 0) \leftrightarrow 0$. The particular ordering of the other elements of $J(1,0)$ is not important as long as the ordering which is chosen is used consistently. Similarly, we put the elements of $J(1,1)$ in a one-to-one correspondence with the integers $2^{n-1}, 2^{n-1}+1$, $\cdots, 2^{n}-1$. Now if $j \in J(1)$ and $i$ is the corresponding integer, $j \leftrightarrow i$, we denote $r_{j}$ by $r_{i}$. With this notation, Eq. (3.4) can be written

$$
u^{i}(t)=a_{-} p_{0} \sum_{i=0}^{N} a_{i} u^{t}\left(t+r_{i}\right)+\int_{t}^{0} W(t-s) u^{t}(s) d s
$$

where we have set $N=2^{n}-1$ and

$$
\begin{aligned}
a_{i} & =C_{j}{ }^{+} / p_{1} \quad \text { if } \quad i \leftrightarrow j \in J(1,0) \\
& =g_{0} C_{j}{ }^{+} / p_{1} \text { if } \quad i \leftrightarrow j \in J(1,1) .
\end{aligned}
$$

Finally, let $[[\tau]]$ denote the greatest integer less than or equal to $\tau$, and let

$$
c\left(k_{1}, k_{2}, \cdots, k_{N}\right)=\frac{\left(k_{1}+k_{2}+\cdots+k_{N}\right) !}{k_{1} ! k_{2} ! \cdots k_{N} !} \text { (multinomial coefficient). }
$$


We now apply [6, Corollary 2] to Eq. (3.8) to obtain

Corollary 2.

$$
u^{t}(t)=\mathfrak{J}_{x,+} u^{i}(t)+\int_{t}^{0} T(t-s) u^{i}(s) d s, \quad-x<t<0
$$

where

$$
\begin{aligned}
J_{x, \pm} v(t)=\left(a_{-} p_{0}\right)^{-1} \sum_{k_{1}=0}^{s_{1}(x)} \sum_{k_{2}=0}^{s_{2}(x)} \ldots \sum_{k_{N}=0}^{s_{N}(x)}\left(-a_{1}\right)^{k_{1}}\left(-a_{2}\right)^{k_{2}} \\
\cdots\left(-a_{N}\right)^{k_{N}} C\left(k_{1}, k_{2}, \cdots, k_{N}\right) v\left(t \pm \sum_{i=1}^{N} k_{i} r_{i}\right),
\end{aligned}
$$

and $s_{1}(x)=\left[\left[x / r_{1}\right]\right]$

$$
s_{i}(x)=\left[\left[\left(x-\sum_{m=1}^{i-1} k_{m} r_{m}\right) / r_{i}\right]\right], \quad 2 \leq i \leq N .
$$

Here, $T$ satisfies

$$
\mathcal{H}^{i} T(t)+\mathfrak{J}_{x,+} W(t)+\int_{t}^{0} T(s) W(t-s) d s=0, \quad-x<t<0 .
$$

We next derive the back-scattering operator or reflection operator by substituting (3.9) into (3.3). This yields

$$
u^{r}(t)=\Re_{x,+} u^{i}(-t)+\int_{-t}^{0} R(s+t) u^{i}(s) d s, \quad 0<t<x,
$$

where, for any $s$

$$
\begin{aligned}
\mathcal{R}_{x, \pm} v(s)= & \sum_{k_{1}=0}^{s_{1}^{(x)}} \sum_{k_{2}=0}^{s_{2}^{(x)}} \cdots \sum_{k_{N}=0}^{s_{N}^{(x)}} K\left(k_{1}, k_{2}, \cdots, k_{N}\right) v\left(s \pm \sum_{i=1}^{N} k_{i} r_{i}\right), \\
K\left(k_{1}, k_{2}, \cdots, k_{N}\right)= & \left(-a_{1}\right)^{k_{1}}\left(-a_{2}\right)^{k_{2}} \cdots\left(-a_{N}\right)^{k_{N}}\left\{-g_{0}\left[C\left(k_{1}, k_{2}, \cdots, k_{N}\right)\right.\right. \\
& -C\left(k_{1}-1, k_{2}, k_{3}, \cdots, k_{N}\right)-C\left(k_{1}, k_{2}-1, k_{3}, \cdots, k_{N}\right) \\
& \left.-\cdots-C\left(k_{1}, k_{2}, \cdots, k_{M}-1, \cdots, k_{N}\right)\right] \\
& +g_{0}{ }^{-1}\left[C\left(k_{1}, k_{2}, \cdots, k_{M}, k_{M+1}-1, k_{M+2}, \cdots, k_{N}\right)\right. \\
& \left.\left.+\cdots+C\left(k_{1}, k_{2}, \cdots, k_{N-1}, k_{N}-1\right)\right]\right\}
\end{aligned}
$$

and we define $M=(N-1) / 2$ and $C\left(\alpha_{1}, \alpha_{2}, \cdots, \alpha_{N}\right)=0$ if any

$\alpha_{i}<0$. Furthermore, $R$ satisfies the relation

$$
R(t)=\operatorname{HC}^{r} T(-t)+\mathfrak{J}_{x,-} V(t)+\int_{-t}^{0} V(t+s) T(s) d s, \quad 0<t<x
$$

where we define $T(t)=V(-t)=0$ if $t>0$.

Notice that if at least one of the $k_{i}$ 's is nonzero, then

$$
\begin{aligned}
C\left(k_{1}, \cdots, k_{N}\right)= & C\left(k_{1}-1, k_{2}, \cdots, k_{N}\right)+C\left(k_{1}, k_{2}-1, k_{3}, \cdots, k_{N}\right) \\
& +\cdots+C\left(k_{1}, \cdots, k_{N}-1\right)
\end{aligned}
$$


and so we can write

$$
\begin{aligned}
K\left(k_{1}, \cdots, k_{N}\right)= & \left(-a_{1}\right)^{k_{1}} \cdots\left(-a_{N}\right)^{k} \cdot\left(g_{0}{ }^{-1}-g_{0}\right)\left[C\left(k_{1}, \cdots, k_{N}\right)\right. \\
& \left.-C\left(k_{1}-1, k_{2}, \cdots, k_{N}\right)-\cdots-C\left(k_{1}, \cdots, k_{M}-1, \cdots, k_{v}\right)\right] .
\end{aligned}
$$

It follows that if $k_{i}=0$ for $M+1 \leq i \leq N$ and at least one $k_{i}$ is nonzero tor $1 \leq i \leq M$, then $K\left(k_{1}, \cdots, k_{N}\right)=0$. Furthermore, certain other terms in $\mathcal{R}_{x,+} u^{i}(-t)$ also vanish. For example, the coefficient of $u^{i}\left(-t+2 l-2 x_{2}+2 x_{1}\right)$ sums to zero.

As we now show, the vanishing of certain terms in the reflection operator is to be expected, and means that we can sense the location of the discontinuities within the medium. To see this, assume $u^{i \prime}$ is continuous everywhere except at $0, u^{i \prime}\left(0^{-}\right) \neq 0$. Then, since this discontinuity can only propagate along characteristics $x \pm t=$ constant, it follows that only certain discontinuities in the derivative of the reflected wave $u^{r \prime}$ are possible, namely, those which correspond to physically attainable reflections within the medium. For example, looking at the situation pictured in Fig. 1, we see that the first reflected discontinuity locates the leading edge of the slab, the second reflected discontinuity locates $x_{1}$, the third locates $x_{2}$, the fourth can be shown to be a second reflections off of $x_{1}$, and the fifth locates $x_{3}=l$.

We now derive a result analogous to [5, Lemma 5]. For convenience, we define $M_{-}{ }^{k}(x$, $t, 0)=0$ if $|t|>x$ and set $M_{-}{ }^{k}(x, t, 0)=M^{k, x}(t)$. Also, for any integer $k, 0 \leq k \leq n-1$ and $i=0$ or 1 , define

$$
\mathscr{L}_{k, i} v(x \pm t)=\left(c_{0} c_{1} \cdots c_{k}\right)^{-1} \sum_{j \in J i k, i} \tilde{C}_{j}^{ \pm} v\left(\tilde{r}_{j}-2 i x+x \pm t\right)
$$

LEMMA 3. If $x_{k}<x<x_{k+1}$ for any integer $k, 0 \leq k \leq n-1$, then

$$
\begin{aligned}
M_{+}{ }^{k}(x, t, 0) & =\mathscr{L}_{k, 1} R(x+t) \\
& +G(x)\left[\mathcal{L}_{k, 0} R(x+t)-\mathfrak{R}_{2 x,+} M^{k, x}(-t)-\int_{-t}^{x} R(s+t) M^{k, x}(s) d s\right], \\
& -x<t<x .
\end{aligned}
$$

Proof: If we use $v=u^{i}, w=u^{r}$ as the Cauchy data in Corollary 1 , then it follows by causality that $u(x, t)=0$ if $t<x$. We now use the reflection operator to express $u^{r}$ in terms of $u^{i}$ in (2.7). Thus, for $t<x$ we arrive at a delay Volterra equation for $u^{i}$ of the type considered in [6]. By the uniqueness result of [6, Corollary 1], it follows (since $u^{i}$ is arbitrary) that the kernel in this integral equation must be identically zero, and our result follows.

Finally, we develop an expression for $G(x)$ in terms of $M_{-}{ }^{k}(x, t, 0)$.

LemmA 4. If $x_{k}<x<x_{k+1}$ for any integer $k, 0 \leq k \leq n-1$, then

$$
\begin{aligned}
G(x)^{-1}= & \alpha_{k}(x)\left\{\beta_{k}+\int_{-x}^{x} \mathfrak{L}_{k, 0} R(x+t) d t-\int_{-x}^{x} M^{k, x}(t)\left[1+\mathcal{R}_{2 x,-} \theta(x+t)\right.\right. \\
& \left.\left.+\int_{-t}^{x} R(s+t) d s\right] d t\right\}
\end{aligned}
$$




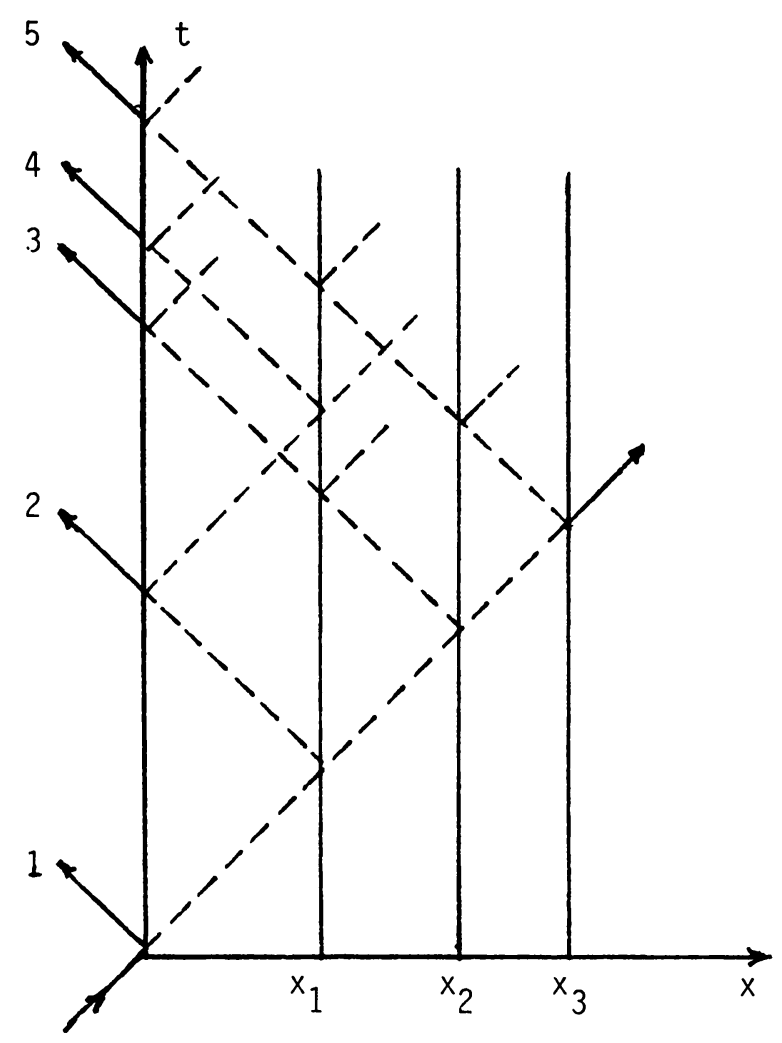

FIG. 1. Pattern of reflected discontinuities.

where

$$
\begin{aligned}
& \alpha_{k}(x)=\left(\sum_{j \in \tilde{J}(k, 0)} \tilde{C}_{j}^{+}-\sum_{j \in \tilde{J}(k, 1)} \tilde{C}_{j}^{-}-\int_{-x}^{x} \mathscr{L}_{k, 1} R(x+t) d t\right)^{-1}, \\
& \beta_{k}=\sum_{j \in \tilde{J}(k, 0)} \tilde{C}_{j}^{-}-\sum_{j \in \tilde{J}(k, 1)} \tilde{C}_{j}^{+} .
\end{aligned}
$$

Proof: Set $v \equiv 1, w \equiv-1$ in Corollary 1. It follows by uniqueness that the left-hand side of Eq. (2.7) is identically zero. Now use Lemma 3 to express $M_{+}{ }^{k}$ in terms of $M_{-}{ }^{k}$, and the proof is completed.

4. The inverse problem. In this section we present our main result (Theorem 3), an integral equation whose solution leads directly to the coefficients of Eq. (1.1), We begin with

LEMma 5. For any integer $k, 0 \leq k \leq n-1$, and for all $x, t$ we have

$$
\exp \left\{\frac{1}{2} \int_{x}^{0}(A(s)-B(s)) d s\right\} \mathscr{L}_{k} \circ \mathcal{F}^{i} u^{t}(x-t)
$$




$$
\begin{aligned}
& +\exp \left\{\frac{1}{2} \int_{x}^{0}(A(s)+B(s)) d s\right\} \mathscr{L}_{k} \circ \mathfrak{H}^{r} u^{t}(-x-t) \\
& =\exp \left\{\frac{1}{2} \int_{x}^{l}(A(s)-B(s) d s\} \mathcal{H}_{k+1} \circ \mathcal{H}_{k+2} \circ \cdots \circ \mathcal{H}_{n} u^{t}(x-t)\right.
\end{aligned}
$$

where $\mathscr{L}_{k}=\left(c_{0} c_{1} \cdots c_{k}\right)^{-1} \mathfrak{K}_{k} \circ \mathfrak{K}_{k-1} \circ \cdots \circ \mathfrak{K}_{0}$.

Proof: Using Lemma 2, our problem is reduced to showing that

$$
\mathfrak{H}^{i} u^{t}(x-t)+G(x) \mathfrak{H}^{r} u^{t}(x+t)=a_{-} \mathcal{H}_{0} \circ \mathcal{H}_{1} \circ \cdots \circ \mathcal{H}_{n} u^{t}(x-t)
$$

Now the right-hand side of $(4.1)$ can be written

$$
\begin{aligned}
a_{-} \mathcal{H}_{0} & {\left[\sum_{j \in J(1,0)} C_{j}{ }^{+} u^{t}\left(r_{j}+x-t\right)-G(x) \sum_{j \in J(1,1)} C_{j}+u^{t}\left(r_{j}-x-t\right)\right] } \\
= & \frac{1}{2} a_{-}\left(c_{0}+1\right)\left[\sum_{j \in J(1,0)} C_{j}^{+} u^{t}\left(r_{j}+x-t\right)-G(x) \sum_{j \in J(1,1)} C_{j}^{+} u^{t}\left(r_{j}-x-t\right)\right] \\
& -\frac{1}{2} a_{-}\left(c_{0}-1\right)\left[G(x) \sum_{j \in J(1,0)} C_{j}^{+} u^{t}\left(r_{j}-x-t\right)-\sum_{j \in J(1,1)} C_{j}^{+} u^{t}\left(r_{j}+x-t\right)\right] .
\end{aligned}
$$

But, as can be seen from (3.1) and (3.2), this is the same as the left-hand side of (4.1) and our result follows.

In the following theorem we use the notation

$$
\begin{aligned}
& F_{k}(x, t)= \alpha_{k}(x)\left[\beta_{k}+\int_{-x}^{x} \mathcal{L}_{k, 0} R(x+s) d s\right] f_{k, 0}(x, t)+f_{k, 1}(x, t), \\
& G_{k}(x, t, s)=-\alpha_{k}(x)\left[1+\mathcal{R}_{2 x,-} \theta(x+s)+\int_{-s}^{x} R(s+y) d y\right] f_{k, 0}(x, t) \\
&+\frac{1}{2} a_{-} \sum_{j \in J(1)} C_{j}^{+} \gamma_{j} \theta\left(r_{j}+x-t\right) \theta\left(x+t-r_{j}\right) R\left(s+t-r_{j}\right) \\
&+\sum_{k_{1}=0}^{s_{1}(2 x)} \sum_{k_{2}=0}^{s_{2}(2 x)} \cdots \sum_{k_{N}=0}^{s_{j}^{(2 x)}} \\
& \cdot K\left(k_{1}, k_{2},\right.\left.\cdots, k_{N}\right) \theta\left(x+s-\sum_{i=1}^{N} k_{i} r_{i}\right) W\left(\sum_{i=1}^{N} k_{i} r_{i}-s-t\right) \\
&+\int_{-s}^{x} R(s+y) W(y-t) d y-V(s+t), \\
& \gamma_{j}=\left(c_{0}+1\right) \text { if } j \in J(1,0) \\
& \gamma_{j}=\left(c_{0}-1\right) \text { if } j \in J(1,1)
\end{aligned}
$$

and, for $i=0$ or 1

$$
\begin{aligned}
f_{k, i}(x, t)= & \mathfrak{L}_{k, i} W(x-t)+\mathfrak{L}_{k, i} V(x+t)-\int_{-x}^{x} W(s-t) \mathscr{L}_{k, i} R(x+s) d s \\
& -\frac{1}{2} a_{-} \sum_{j \in J(1)} C_{j}{ }^{+} \gamma_{j} \theta\left(r_{j}+x-t\right) \mathfrak{L}_{k, i} R\left(x+t-r_{j}\right) .
\end{aligned}
$$

Here, $\mathcal{L}_{k, i} R(x+s) \equiv\left(\left.\mathfrak{L}_{k, i} R(x+t)\right|_{t=s}\right.$. 
THEOREM 3. For any integer $k, 0 \leq k \leq n-1$ and any $x, x_{k}<x<x_{k+1}$ we have

$$
\begin{aligned}
\mathcal{F C}^{r} M^{k, x}(t-2 l)-\mathcal{H}^{i}\left\{\theta(x+t-2 l) \mathbb{R}_{2 x,+} M^{k, x}(t-2 l)\right\} \\
=F_{k}(x, 2 l-t)+\int_{-x}^{x} M^{k, x}(s) G_{k}(x, 2 l-t, s) d s, \quad-x<t<x
\end{aligned}
$$

where $\mathfrak{H C}^{i}$ acting on $\theta(x+t-2 l) \mathcal{R}_{2 x,+} M^{k, x}(t-2 l)$ shifts the arguments in both functions.

Proof: We fix $x, x_{k}<x<x_{k+1}$, and express $u(x, t)$ in terms of $u^{t}$ by using Theorem 1, and also express $u(x, t)$ in terms of $u^{i}, u^{r}$ by using Corollary 1 . Equating these expressions, we then use Theorem 2 to write $u^{i}, u^{r}$ in terms of $u^{t}$. In the resulting equation the terms not involving integrals can be shown to cancel out by means of Lemma 5. Setting $t=x$ in the remaining integrals yields an expression of the form $\int_{-x} x(\cdot) u^{t}(y-x) d y=0$, which, by virtue of the fact that $u^{t}$ is arbitrary, shows that the portion of the integrand in parentheses is identically zero. (This is in fact a restatement of the causality result of Lemma 3 , but in a more complicated form.) Now using the fact that the integrals of the form $\int_{-x}^{x}(\cdot) u^{t}(y-t) d y$ are identically zero, we set $t=2 l-x$ and, by the same reasoning as above, conclude that the integral $\int_{x}^{2 l-x}(\cdot) u^{t}(y-t) d y$ is identically zero because its integrand is zero. Finally considering $2 l-x<t<2 l+x$ and using the fact that $u^{t}$ is arbitrary yields an integral equation involving $M_{ \pm}{ }^{k}(x, y, 0)$. Using Lemma 3 to eliminate $M_{+}{ }^{k}(x, y, 0)$ and Lemma 4 to eliminate $G(x)$ and replacing the variable $t$ with $2 l-t$ yields Eq. (4.2) and completes the proof.

Although the integral equation (4.2) looks quite formidable in its most general form, it simplifies considerably when considering a specific problem. (We shall demonstrate this presently.) In some cases this equation can be reduced to a Fredholm equation of the second kind (for $x$ fixed) by using the technique in [6]. In other cases, because of the presence of both advanced and delayed arguments, this reduction may not be possible. However, we present in this section a numerical method of solution which is applicable in either case.

Upon solving Eq. (4.2) for $M_{-}{ }^{k}(x, t, 0),-x<t<x, x_{k}<x<x_{k+1}$, we then apply Lemma 4 to obtain $G(x)$ for $x_{k}<x<x_{k+1}$. We next determine $B(x)$ on the same interval from the relation $B=-G^{\prime} / G$. Finally, we obtain the quantity $C-A^{\prime} / 2-A^{2} / 4$ for $x_{k}<x$ $<x_{k+1}$ from Eq. (2.8). Doing this for all subintervals $\left(x_{k}, x_{k+1}\right)$ completes the solution of the inverse problem. Notice that it is not necessary to solve for $M_{-}{ }^{0}, M_{-}{ }^{1}, \cdots$ consecutively.

Looking at (4.2) it is readily determined that the data required for the construction of that integral equation can be derived from the following items:

(a) Reflection kernel $R(s), 0<s<4 l$,

(b) Transmission kernel $T(s),-4 l<s<0$,

(c) Constants $a_{-} p_{0}, g_{n} G\left(x_{n}\right)^{-1}, x_{n}$ and $c_{i}, x_{i}, G\left(x_{i}\right), i=0,1, \cdots, n-1$.

For example, knowing $T(s)$, we can determine $W(s)$ from (3.10) using the results from [6] on delay Volterra equations. We similarly obtain $V(s)$ from (3.13).

Observe that we are required to know $T(s)$ for $-4 l<s<0$, whereas for the problem in [5] we only needed the transmission kernel for $-2 l<s<0$. The difference here occurs in solving Eq. (3.13) for $V$. For the special case in [5] the resolvent kernel for the analogous equation is $W$, while for the present problem $W$ is not the resolvent kernel.

As indicated in the introduction, the data $(a),(b),(c)$ can be determined from $u^{i}(s)$, $u^{t}(s), u^{r}(-s)$ for $-4 l<s<0$ if hypotheses $(f),(g),(h)$ of Sec. 1 are satisfied. This can 
be done in much the same way as indicated in [5, Section 2]. The constants in item (c) are most easily derived from the coefficients of the shift operators $R_{2 l+,+}, J_{2 l+,+}$ and from the corresponding shifts (see Sec. 5). The kernels $R, T$ are obtained from Volterra integral equations as shown in [5].

The nature of the integral equation (4.2) can be determined by examining the terms on the left-hand side of that equation. We do this for the case of a single discontinuity at $x_{1}$ (and, of course, discontinuities at $x_{0}=0, x_{2}=l$ ). We restrict our attention to the case $x_{1}<$ $l / 2$, the case $x_{1}>l / 2$ being similar to what is done here. For $0<x<x_{1}$ the left-hand side of Eq. (4.2) reduces to

$$
d_{0} M_{-}{ }^{\circ}(x, t, 0)
$$

where

$$
d_{k}=-a_{+} c_{0}\left(c_{1}+1\right)\left(c_{2}-1\right)\left[-g_{0} g_{1} G\left(x_{1}\right)^{-1 / 4}\right]^{k} /\left(2\left(c_{0}+1\right)\right),
$$

and so (4.2) is a Fredholm equation of the second kind. For $x_{1}<x<l-x_{1}$ the left-hand side of (4.2) becomes

$$
\sum_{k=0}^{s(x)} f_{k}(t) M_{-}{ }^{1}\left(x, t+2 k x_{1}, 0\right)
$$

where $s(x)=\left[\left[x / x_{1}\right]\right]$,

$$
f_{k}(t)=d_{k}\left[1-\theta\left(x+t-2 x_{1}\right) g_{1}{ }^{2} / 4\right], \quad 0 \leq k \leq s(x)-1, \quad f_{s(x)}(t)=d_{s(x)} .
$$

It follows that in this case (4.2) can be reduced to a delay equation of the form considered in [6], and is therefore equivalent to a Fredholm equation of the second kind. Finally, for $l-x_{1}<x<l$ we obtain

$$
\sum_{k=0}^{s(x)} f_{k}(t) M_{-}{ }^{1}\left(x, t+2 k x_{1}, 0\right)+\sum_{k=0}^{s s(x)} h_{k}(t) M_{-}{ }^{1}\left(x, t-2 l+2(k+1) x_{1}, 0\right)
$$

where

$$
h_{k}(t)=d_{k} g_{1} g_{2}^{-1} G\left(x_{1}\right)^{-1} G(l) \theta\left(x+t+2 x_{1}-2 l\right), \quad 0 \leq k \leq s(x) .
$$

Thus, the integral equation contains both advanced and delayed arguments. However, for this particular case notice that for $-x<t<2 x_{1}-x$, Eq. (4.2) is

$$
\sum_{k=0}^{s_{1} x_{1}} d_{k} M_{-}{ }^{1}\left(x, t+2 k x_{1}, 0\right)=F_{1}(x, 2 l-t)+\int_{-x}^{x} M_{-}{ }^{1}(x, s, 0) G_{1}(x, 2 l-t, s) d s .
$$

It follows that

$$
\begin{array}{r}
\sum_{k=0}^{s \mid x} d_{k} M_{-}{ }^{1}\left(x, t-2 l+2(k+1) x_{1}, 0\right)=F_{1}\left(x, 4 l-2 x_{1}-t\right) \\
+\int_{-x}^{x} M_{-}{ }^{1}(x, s, 0) G_{1}\left(x, 4 l-2 x_{1}-t, s\right) d s, \quad 2 l-2 x_{1}-x<t<x .
\end{array}
$$

Therefore, our integral equation for $l-x_{1}<x<l$ can be rewritten as

$$
\begin{aligned}
& \sum_{k=0}^{s(x)} f_{k}(t) M_{-}{ }^{1}\left(x, t+2 k x_{1}, 0\right) \\
& \quad=F_{1}(x, 2 l-t)-g_{1} g_{2}{ }^{-1} G\left(x_{1}\right)^{-1} G(l) F_{1}\left(x, 4 l-2 x_{1}-t\right) \theta\left(x+t+2 x_{1}-2 l\right)
\end{aligned}
$$




$$
\begin{aligned}
& +\int_{-x}^{x} M_{-}{ }^{1}(x, s, 0)\left[G_{1}(x, 2 l-t, s)\right. \\
& \left.-g_{1} g_{2}{ }^{-1} G\left(x_{1}\right)^{-1} G(l) G_{1}\left(x, 4 l-2 x_{1}-t, s\right) \theta\left(x+t+2 x_{1}-2 l\right)\right] d s
\end{aligned}
$$

and this equation is again reducible to a Fredholm equation of the second kind.

Although in the more general case the above reduction is not always possible, we now show that it is in fact quite straightforward to set up a system of equations for the numerical solution of (4.2). This is done in a manner similar to that for a Fredholm equation [3]. We begin by fixing $k, 0 \leq k \leq n-1$. For a fixed $x, x_{k}<x<x_{k+1}$, we assume we can choose a grid size $d$ such that $2 x_{i}$ is an integral multiple of $d, 1 \leq i \leq n$, and $2 x / d=$ $m$, an integer. This can be done, for example, if the $x_{i}$ 's and $x$ are rational numbers. Now set

$$
M_{p}=M_{-}{ }^{k}(x,-x+p d, 0), \quad p=0,1, \cdots, m .
$$

We choose a quadrature formula

$$
\int_{-x}^{x} M_{-}{ }^{k}(x, s, 0) G_{k}(x, 2 l-t, s) d s \cong \sum_{p=0}^{m} z_{p}(t) M_{p}
$$

where the $z_{p}(t)$ 's are known. Using (4.3), (4.4) in (4.2) and evaluating at $t=-x,-x+d$, $-x+2 d, \cdots-x+m d$ we arrive at a system of $(m+1)$ linear algebraic equations for the $(m+1)$ unknowns $M_{0}, M_{1}, \cdots, M_{m}$.

5. The scattering data. It was mentioned in Sec. 4 that the data in item $(c)$ could be determined from the shift opeartors $R_{2 l+,+}, J_{2 l+,+}$. We now demonstrate this. Assuming $u^{i \prime}(s)$ is continuous everywhere except at $s=0, u^{i \prime}(0-)=\delta \neq 0$, it follows from hypothesis $(g)$ in Sec. 1 that the reflected discontinuities in $u^{r \prime}$ specify the number and location of the points $x_{i}$ in consecutive order, $i=0,1, \cdots, n$. Specifically, $2 x_{i}$ is the time interval between the initial discontinuity in $u^{r^{\prime}}\left(u^{r^{\prime}}(0+)\right)$ and the discontinuity in $u^{r^{\prime}}$ at the point $2 x_{i}\left(\left[u^{r \prime}(t)\right]^{t-2 x_{1}{ }^{+}} t-2 x_{1}-\right)$. In this way it can be determined whether a discontinuity in $u^{r \prime}$ is due to multiple internal reflections off of previously located interfaces $x_{i}$, or is due to a new point of discontinuity $x_{j}$. Hypothesis $(h)$ of Sec. 1 implies that after a finite time we need look for no additional discontinuities.

To determine the other required constants, first notice that since $u^{r^{\prime}}(0+)=-g_{0} \delta$ and $u^{t \prime}(0-)=\left(a_{-} p_{0}\right)^{-1} \delta$, it follows that $c_{0}$ and $a_{-} p_{0}$ are known. To obtain the remaining constants it is easiest to use the transmitted wave. Knowing the $x_{i}$ 's, we can associate discontinuities in $u^{t \prime}$ with internal reflections off of specific interfaces by using hypothesis $(g)$ and the fact that the velocity of propagation is unity. We introduce the notation

$$
\begin{gathered}
D_{i}^{ \pm}=g_{i} G\left(x_{i}\right)^{ \pm 1}, \quad i=0,1, \cdots, n \\
E_{i}=D_{i}{ }^{-} D_{i-1}{ }^{+}, \quad i=1,2, \cdots, n \\
F_{i}=D_{i}{ }^{-} D_{i-2}{ }^{+}-2 E_{i} E_{i-1}, \quad i=2,3, \cdots, n .
\end{gathered}
$$

Now from (3.9) it can be shown that

$$
\left[u^{\prime \prime}(-t)\right]^{t=\left(2 x_{i}-2 x_{i-1}\right)+} t=\left(2 x_{i}-2 x_{i-1}\right)-=-\left(a_{-} p_{0}\right)^{-1} E_{i} \delta, \quad i=1,2, \cdots, n
$$

and 


$$
\left[u^{\prime \prime}(-t)\right] \quad t=\left(2 x_{i}-2 x_{i-1}\right)+{ }_{t=\left(2 x_{i}-2 x_{i-1}\right)-}-\left(a_{-} p_{0}\right)^{-1} F_{i} \delta, \quad i=2,3, \cdots, n
$$

and so the $E_{i}$ 's and $F_{i}$ 's are known (i.e. can be determined by observing the transmitted wave). Since $g_{0}$ is also known, it follows from (5.1) that $D_{1}^{-}$is known, and from (5.2) that $D_{2}{ }^{-}$is known. We next obtain $D_{1}^{+}$from (5.1), then $D_{3}^{-}$from (5.2), $D_{2}^{+}$from $(5.1), D_{4}^{-}$ from (5.2), $D_{3}{ }^{+}$from (5.1), etc. In this way we obtain $D_{i}{ }^{+}, D_{i}{ }^{-}$for $i=1,2, \cdots, n-1$ and $D_{n}{ }^{-}$. It follows that all of the required data has been determined.

There are other methods for determining this data. For example, the coefficients of the reflection operator can be of some use. These are somewhat more unwieldy than the transmission operator coefficients, but we list the first few here to show the nature of (3.12). Letting $h_{i}=1-g_{i}{ }^{2}$, we have

$$
\begin{aligned}
& {\left[u^{r \prime}(t)\right]^{t=2 k x_{1}{ }^{+}}{ }_{t=2 k x_{1^{-}}}=h_{0}\left(-D_{1^{-}}\right)^{k} \delta, \quad k=1,2, \cdots,} \\
& {\left[u^{r \prime}(t)\right]^{t=2 x_{2^{+}}+} t=2 x_{2^{-}}=-h_{0} h_{1} D_{2}^{-} \delta,} \\
& {\left[u^{r \prime}(t)\right]^{t=4 x_{2}{ }^{+}}{ }_{t=4 x_{2^{-}}}=h_{0} h_{1}\left(1-3 g_{1}{ }^{2}\right)\left(D_{2}{ }^{-}\right)^{2} \delta \text {, }}
\end{aligned}
$$

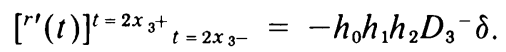

Notice that in using (3.11) to derive the third equation, the coefficient of $u^{i}\left(-t+4 x_{2}\right)$ is the sum of three terms using different sets of values of the $k_{i}$ 's. This follows from the fact that $\sum_{i=1}^{N} k_{i} r_{i}=4 x_{2}$ can be written in three ways:

$$
2\left(2 x_{2}\right), 2 x_{2}+\left(2 x_{2}-2 x_{1}\right)+2 x_{1}, 2\left(2 x_{2}-2 x_{1}\right)+2\left(2 x_{1}\right) .
$$

If hypothesis $(g)$ of Sec. 1 is not satisfied, the reflected and transmitted discontinuities will interfere with each other and it may not always be possible to associate a jump in $u^{r \prime}$ or $u^{t \prime}$ with a specific set of coefficients of $R_{2 l+,+}$ or $J_{2 l+,+}$. In fact, it appears possible that discontinuities can remain hidden. However, this depends on the specific geometry of the problem. There are cases in which hypothesis $(g)$ is not satisfied but the required data can still be obtained from scattered waves.

6. The electromagnetic inverse problem with piecewise constant conductivity and permittivity. We now show that the previous results can be easily used to reconstruct the conductivity and permittivity profiles for the case in which it is known a priori that these functions are piecewise constant. This corresponds to the case in which the medium is composed of a stack of conducting plates of different (unknown) thicknesses, each plate having constant electric properties. Thus, we consider Eq. (1.2) where

$$
\begin{aligned}
\epsilon(z) & =\epsilon_{i} \text { for } z_{i-1}<z<z_{i}, \quad i=1,2, \cdots, n \\
& =\epsilon_{0} \text { otherwise } \\
\sigma(z) & =\sigma_{i} \text { for } z_{i-1}<z<z_{i}, \quad i=1,2, \cdots, n \\
& =0 \text { otherwise }
\end{aligned}
$$

and $E, E_{z}$ are continuous across the interfaces $z=z_{i}$. Under the change of variables (1.3) we obtain (1.1) with $A \equiv C \equiv 0$ and $B(x)=-\sigma(z) / \epsilon(z)$. As indicated in Sec. 5 , the locations of the discontinuities in the derivative of the reflected wave yield the constants $c_{0}$ and $x_{i}, 1 \leq i \leq n$ (provided hypothesis $(g)$ is satisfied). The strengths of the discontinuities in the derivative of the transmitted wave yield the constants $c_{i}, G\left(x_{i}\right)$ for $1 \leq i \leq n-1$ and $g_{n} G\left(x_{n}\right)^{-1}$. Notice that, physically, $x_{i}$ denotes the propagation time between $z=0$ and $z=$ $z_{i}$. 
Since $c_{i}=\epsilon_{i+1} / \epsilon_{i}$ for $0 \leq i \leq n$ (with $\epsilon_{n+1}=\epsilon_{0}$ ) it now follows that the permittivities $\epsilon_{1}$, $\epsilon_{2}, \cdots, \epsilon_{n}$ are known. Thus, $c_{n}$ is also known and so is $G\left(x_{n}\right)$. Furthermore, since

$$
x_{i}-x_{i-1}=\left(\epsilon_{i} \mu_{0}\right)^{1 / 2}\left(z_{i}-z_{i-1}\right), \quad 1 \leq i \leq n
$$

we can now determine $z_{1}, z_{2}, \cdots, z_{n}$ consecutively. Finally,

$$
\sigma_{i}=\left(\epsilon_{i} / \mu_{0}\right)^{1 / 2}\left(z_{i}-z_{i-1}\right) \ln \left[G\left(x_{i}\right)^{-1} G\left(x_{i-1}\right)\right], \quad 1 \leq i \leq n .
$$

This completes the solution of the inverse problem. We see that in this special case it is not necessary to solve an integral equation.

\section{REFERENCES}

[1] A. A. Buzdin, On an inverse problem in a medium with absorption, Proc. Steklov Inst. Math. 115, 77-85 (1971)

[2] M. Jaulent, Inverse scattering problems in absorbing media, J. Math. Phys. 17, 1351-1360 (1976)

[3] L. Kantorovich and V. Krylov, Approximate methods of higher analysis, Noordhoff, 1964

[4] I. Kay, The inverse problem when the reflection coefficient is a rational function, Comm. Pure Appl. Math. 13, $371-393(1960)$

[5] R. Krueger, An inverse problem for a dissipative hyperbolic equation with discontinuous coefficients, Quart. Appl. Math. 34, 129-147 (1976)

[6] R. Krueger, Some results on linear delay integral equations, J. Math. Anal. Appl. (to appear)

[7] M. Roseau, Asymptotic wave theory, North-Holland, 1976

[8] V. Weston, On the inverse problem for a hyperbolic dispersive partial differential equation, J. Math. Phys. 13, 1952-1956 (1972)

[9] V. Weston, On inverse scattering. J. Math. Phys. 15, 290-213 (1974)

[10] V. Weston and R. K rueger, On the inverse problem for a hyperbolic dispersive partial differential equation, II, J. Math. Phys. 14, 406-408 (1973); 14, 1296 (1973) 